\title{
Microbial and metabolomic profiles in correlation with depression and anxiety co-morbidities in diarrhoea-predominant IBS patients
}

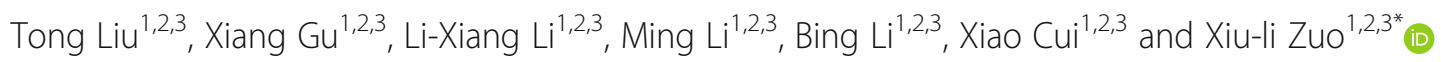

\begin{abstract}
Background: Psychological co-morbidities in irritable bowel syndrome (IBS) have been widely recognized, whereas less is known regarding the role of gut microbial and host metabolic changes in clinical and psychological symptoms in IBS.

Results: A total of 70 diarrhoea-predominant IBS (IBS-D) patients and 46 healthy controls were enrolled in this study. Stool and urine samples were collected from both groups for 165 rRNA gene sequencing and metabolomic analysis.

The results showed that fecal microbiota in IBS-D featured depleted Faecalibacterium (adjusted $P=0.034$ ), Eubacterium rectale group (adjusted $P=0.048$ ), Subdoligranulum (adjusted $P=0.041$ ) and increased Prevotella (adjusted $P=0.041$ ). O-ureido-L-serine, 3,4-dihydroxybenzenesulfonic acid and (R)-2-Hydroxyglutarate demonstrated lower urinary concentrations in IBS-D patients. We further built correlation matrices between gut microbe abundance, differentiated metabolite quantities and clinical parameters. Dialister manifested negative association with IBS severity $(r=-0.285, P=0.017)$, anxiety $(r=-0.347, P=0.003)$ and depression level $(r=-0.308, P=0.010)$. Roseburia was negatively associated with IBS severity $(r=-0.298, P=0.012)$. Twenty metabolites correlated with anxiety or depression levels, including 3,4-dihydroxymandelaldehyde with SAS $(r=-0.383, P=0.001)$, 1methylxanthine with SDS $(r=-0.347, P=0.004)$ and 1D-chiro-inositol with SAS $(r=-0.336, P=0.005)$. In analysis of microbe-metabolite relationship, 3,4-dihydroxymandelaldehyde and 1-methylxanthine were negatively correlated with relative abundance of Clostridium sensu stricto.
\end{abstract}

Conclusions: Our findings demonstrated altered microbial and metabolomic profiles associated with clinically and psychological symptoms in IBS-D patients, which may provide insights for further investigations.

Keywords: Irritable bowel syndrome, Depression, Anxiety, Microbial community, Metabolomics

\footnotetext{
* Correspondence: zuoxiuli@sdu.edu.cn

'Department of Gastroenterology, Qilu Hospital, Cheloo College of Medicine,

Shandong University, 107 Wenhuaxi Road, Jinan 250012, Shandong Province, China

2Laboratory of Translational Gastroenterology, Qilu Hospital, Cheloo College of Medicine, Shandong University, Jinan, China

Full list of author information is available at the end of the article
}

(c) The Author(s). 2020 Open Access This article is licensed under a Creative Commons Attribution 4.0 International License, which permits use, sharing, adaptation, distribution and reproduction in any medium or format, as long as you give appropriate credit to the original author(s) and the source, provide a link to the Creative Commons licence, and indicate if changes were made. The images or other third party material in this article are included in the article's Creative Commons licence, unless indicated otherwise in a credit line to the material. If material is not included in the article's Creative Commons licence and your intended use is not permitted by statutory regulation or exceeds the permitted use, you will need to obtain permission directly from the copyright holder. To view a copy of this licence, visit http://creativecommons.org/licenses/by/4.0/ The Creative Commons Public Domain Dedication waiver (http://creativecommons.org/publicdomain/zero/1.0/) applies to the data made available in this article, unless otherwise stated in a credit line to the data. 


\section{Background}

Irritable bowel syndrome (IBS), a common psychosomatic disorder characterized by abdominal pain, bloating and altered bowel habits, is the most commonly diagnosed gastroenterology disorder which affects over $10 \%$ of the population globally and the prevalence varies regionally $[1,2]$. Despite the relatively good prognosis, IBS affects the quality of life of patients immensely and extensively. Accumulating evidence demonstrated the basic pathophysiology of IBS is disordered brain-gut interaction, frequently accompanied by motility disturbance, visceral hypersensitivity, mild mucosal inflammation, altered gut microbiota, psychiatric disorders and abnormal central nervous system processing [3].

Psychiatric co-morbidities, including higher level of perceived stress, clinically significant depression and/or anxiety, somatization and altered behavioural pattern, are commonly seen in IBS patients and may either predate or generate the intestinal symptoms [4]. It has been reported that up to $94 \%$ of IBS patients demonstrate psychiatric disorders, including somatoform disorders, major depression and anxiety [5]. Among multiple pathophysiological mechanisms interpreting the psychiatric manifestations in IBS patients, the commensal gut microbial population has emerged to be potentially significant [6]. Using advanced tools like next generation sequencing, researchers have been allowed to obtain a more thorough overview of gut microbial composition and metabolic processes. Some studies have reported a different microbial pattern in IBS patients and animal models compared to their healthy controls, mainly characterized by altered microbial richness, fluctuated abundance of phyla Firmicutes and Bacteroides. However, existing results are still inconsistent $[6,7]$.

More recently, gut microbiota has been proposed to interfere with host state via metabolism-regulating ways $[8,9]$, and efforts have been made to describe a possibly distinct metabolic profile in IBS patients using metabolomic approaches [10, 11]. Additionally, metabolomic tools assist in understanding mechanisms of improving IBS symptoms by treatment like probiotics [12], low fermentable oligo-di-mono-saccharides and polyols (lowFODMAP) diet [13] and non-absorbable antibiotics [14]. Urine is a preferred biomaterial in metabolomic analysis due to its easy accessibility, considerable metabolite concentration and exemption of interfering proteins and lipids compared to serum, faecal extraction and cerebrospinal fluid [15]. However, few studies have combined microbiome and metabolomic data in IBS population, and correlations between microbial, metabolomic changes and psychological alterations in IBS are currently less discussed.

In this study, we hypothesized that diarrhoea-predominant irritable bowel syndrome (IBS-D) patients featured gut microbiota alterations and aberrant metabolism compared with healthy population, which were correlated with IBS bowel symptom and psychological co-morbidity. By detailed analysis of the faecal microbiota and a urinary metabolomic approach, we aimed to characterize those features and further investigate their relationship with IBS-D clinical or psychological status.

\section{Results}

\section{Characteristics of subjects}

Seventy IBS-D patients and 46 healthy controls (HC) were involved in this study. IBS-D duration of patients varied from 6 months to 20 years, with an average \pm SD duration of $3.97 \pm 5.04$ years. No significant differences in gender and age were shown between IBS-D group and HC group. Body mass index (BMI) was well balanced between two groups to decrease its potential effect upon microbial composition and metabolism (Table 1). Bristol stool scale and IBS symptom severity scale (IBS-SSS) reflected significantly severer symptoms in IBS-D group than HC group (Table 1). Several classical psychological scales (see Methods section) were utilised to measure anxiety and depression level of IBS-D patients (Table 1).

\section{Characterization of faecal microbiota in IBS-D patients and HCs}

Stool samples were collected from 70 IBS-D patients and 44 HCs. After data trimming, we obtained an average of 52,298 (ranging from 32,092 to 72,405) reads per sample. The Good's coverage reached over 0.997 in both IBS-D and HC group. Simpson index $(0.099 \pm 0.079$ vs. $0.075 \pm 0.038$, adjusted $P=0.041)$ indicated a higher biodiversity in faeces of HCs (Fig. 1a). Interestingly, Ace

Table 1 Demographic and clinical data of IBS-D patients and $\mathrm{HCS}$

\begin{tabular}{llll}
\hline & IBS-D $(n=84)$ & $\mathrm{HC}(n=46)$ & $P$ value \\
\hline Age, Mean (SD) & $41.76(11.57)$ & $38.30(13.13)$ & 0.139 \\
Sex & & & \\
$\quad$ Female & 24 & 21 & 0.219 \\
$\quad$ Male & 46 & 25 & \\
BMl, Mean (SD) & $23.36(3.49)$ & $23.63(3.76)$ & 0.700 \\
Bristol scale, Mean (SD) & $5.26(0.70)$ & $3.89(0.74)$ & 0.000 \\
IBS-SSS, Mean (SD) $_{\text {Duration, Mean (SD), years }}$ & $286.29(43.14)$ & $120.22(44.04)$ & 0.000 \\
SDS $^{\text {a }}$ & $3.97(5.04)$ & $\mathrm{N}^{f}$ & $\mathrm{~N}$ \\
SAS $^{\text {b }}$ & $49.89(9.59)$ & $\mathrm{N}$ & $\mathrm{N}$ \\
HADS $^{\mathrm{c}}$ & $48.81(9.79)$ & $\mathrm{N}$ & $\mathrm{N}$ \\
HAM-D $^{\mathrm{d}}$ & $16.57(6.58)$ & $\mathrm{N}$ & $\mathrm{N}$ \\
HAM-A $^{\mathrm{e}}$ & $13.23(5.15)$ & $\mathrm{N}$ & $\mathrm{N}$ \\
\hline
\end{tabular}

${ }^{a}$ SDS Self-reported depression scale, ${ }^{\mathrm{b}}$ SAS Self-reported anxiety scale, ${ }^{\mathrm{c}} H A D S$ Hospital Anxiety and Depression Scale, ${ }^{\mathrm{d}} H A M-D$ Hamilton Depression Scale, ${ }^{\mathrm{e}} H A M-A$ Hamilton Anxiety Scale, ${ }^{\mathrm{f}} \mathrm{N}$ no data 


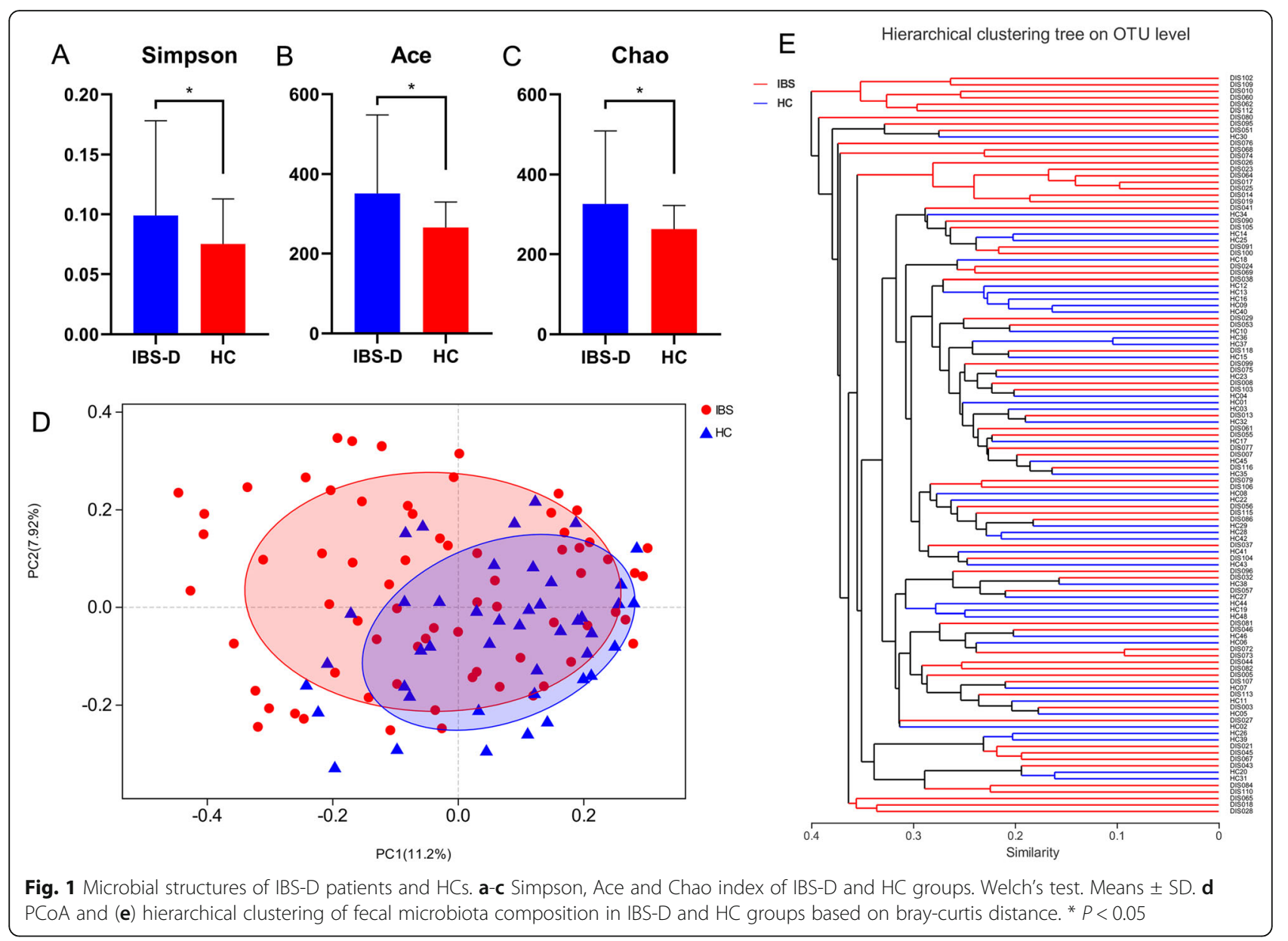

(351.17 \pm 197.79 vs. $265.74 \pm 63.77$, adjusted $P=0.007$ ) and Chao ( $326.29 \pm 183.19$ vs. $263.28 \pm 59.05$, adjusted $P=0.018$ ) reflected that faecal microbial richness in IBS-D patients was higher in comparison of that in HCs (Fig. 1b, c).

Hierarchical clustering and principal co-ordinates analysis (PCoA) based on bray-curtis distances were applied at the level of operational taxonomy unit (OTU) to identify the similarity of microbial community structures between faecal samples from IBS-D patients and HCs (Fig. 1d, e). As shown in Fig. 1e, HC samples were unevenly mixed with IBS-D samples, whereas there were no explicit divisions of two clusters. Similarly, on the PCoA plot, faecal samples from these two groups could not be clearly separated.

Comparisons of faecal microbiota composition between IBS-D and HC group at different taxonomic levels were conducted applying the Mann-Whitney U test. At the phylum level (Fig. 2a), Firmicutes (61.21\%), Bacteroidetes (20.52\%), Proteobacteria (9.71\%) and Actinobacteria $(7.32 \%)$ were the most abundant phyla of IBS-D group, and in $\mathrm{HC}$ group the order was Firmicutes (71.97\%), Bacteroidetes (15.42\%), Actinobacteria (6.82\%) and Proteobacteria (5.48\%). The abundance of the most predominant phyla Firmicutes was significantly decreased in IBS-D patients compared with HCs (adjusted $P=0.033$ ). Among the first 10 predominant phyla, Cyanobacteria (adjusted $P=0.024$ ), Fusobacteria (adjusted $P<0.001$ ) and Chloroflexi (adjusted $P=0.001$ ) were also significantly higher in faecal samples of IBS-D patients though their abundances were lower than $1 \%$ in both groups. Faecalibacterium $(6.02 \% \pm 6.48 \%$ vs. $9.14 \% \pm$ $6.33 \%$, adjusted $P=0.034$ ), Eubacterium rectale group $(4.87 \% \pm 6.83 \%$ vs. $6.48 \% \pm 5.70 \%$, adjusted $P=0.048)$ and Subdoligranulum $(3.20 \% \pm 4.78 \%$ vs. $4.87 \% \pm 4.55 \%$, adjusted $P=0.041$ ) were genera of higher abundances in stool samples of HC group, while Prevotella $9(4.84 \% \pm$ $11.39 \%$ vs. $3.75 \% \pm 8.52 \%$, adjusted $P=0.041)$ was increased in IBS-D group. Bacteroides (adjusted $P=0.826$ ) and Blautia (adjusted $P=0.137$ ) were the most predominant genera in IBS-D and HC group (Fig. 2b), respectively. Yet, the differences of both between 2 groups didn't reach significance after false discovery rate (FDR) correction. Cladogram from phylum to genus level using linear discriminant analysis effect size (LEfSe) demonstrated specific faecal microbial taxa associated with IBS-D or health (Fig. 2c). Most taxa of high linear discriminant analysis 


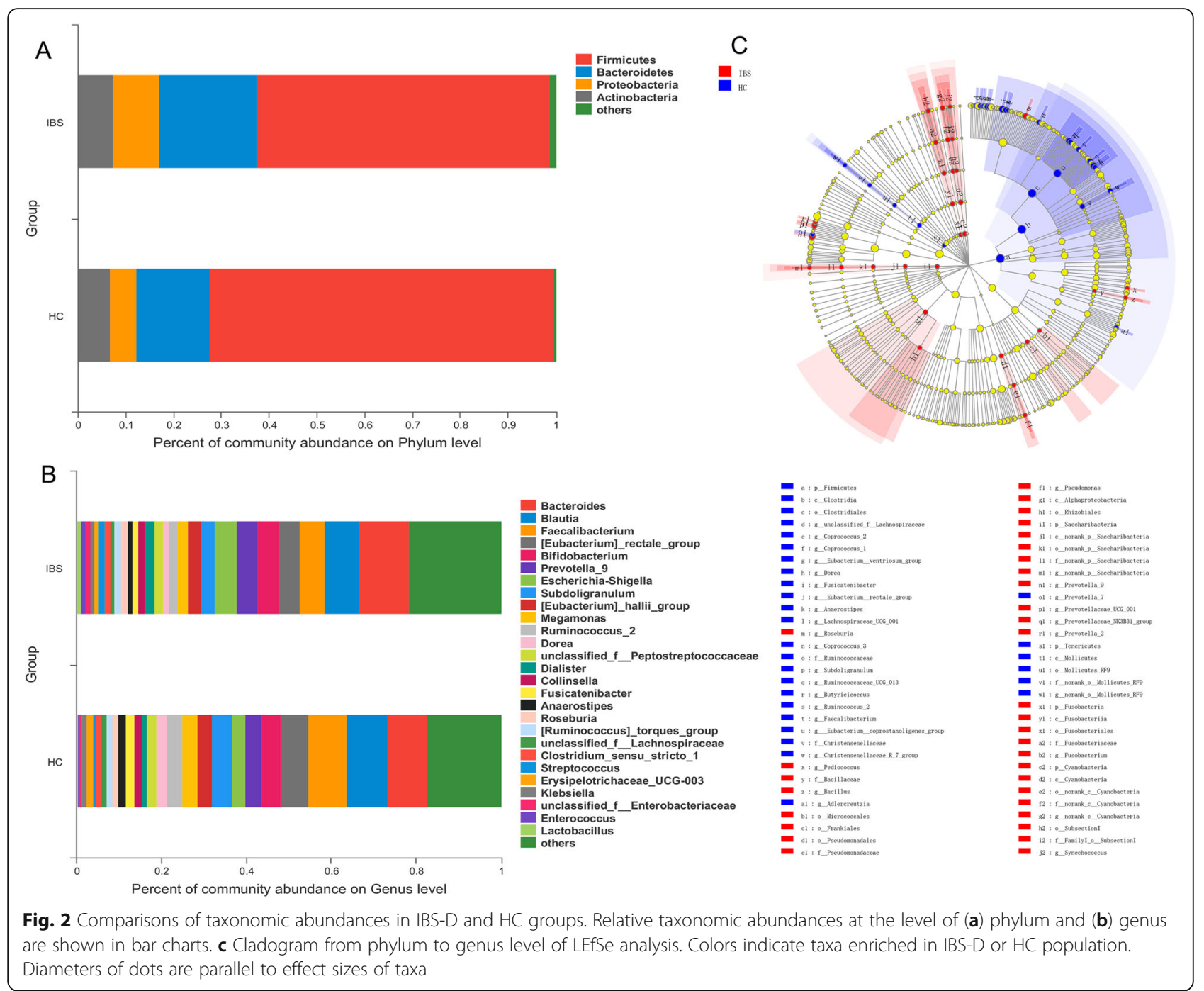

(LDA) scores belonged to the phylum Firmicutes, which had the potential to be identifying biomarkers for IBS-D or HC (Fig. S1).

\section{Associations between clinical and psychological characteristics and differential microbiota composition in IBS-D}

We included six variables regarding clinical symptoms and psychological abnormalities (IBS-SSS, Self-reported Anxiety and Depression Scales (SAS / SDS), Hospital Anxiety and Depression Scale (HADS), Hamilton Anxiety and Depression Scales (HAM-A / HAM-D)) that may be related to microbial signature in 70 IBS-D patients. All variables were included after variance inflation factor analysis at a threshold of 10 to rule out redundant parameters. Canonical correspondence analysis (CCA) demonstrated the extent to which those factors shaped the microbial community in IBS-D (Fig. 3a, Table S1).
All six factor scores significantly correlated with microbial structure (adjusted $P \leq 0.001$ ).

Furtherly, we examined associations between composition of faecal microbiota and those clinical metadata using Spearman's correlation coefficient. Significant correlations were found between relative abundances of genus Roseburia and IBS severity $(r=-0.298, P=0.012)$, Dialister and IBS severity $(r=-0.285, P=0.017)$, Dialister and HAM-D $(r=-0.308, P=0.010)$, Dialister and HAM-A $(r=-0.347, P=0.003)$, Bifidobacterium and SAS $(r=0.276, P=0.021)$, Bifidobacterium and HAMA $(r=0.325, P=0.006)$, Megamonas and HAM-A $(r=$ - 0.235, $P=0.049)$, Streptococcus and HAM-D $(r=$ $0.269, P=0.024)$, Streptococcus and HAM-A $(r=0.262$, $P=0.029)$ (Fig. 3b-f). We then employed the multivariate statistical approach to relate psychological scale parameters to microbial abundances for which age, gender and IBS severity were adjusted considering their confounding effect upon gut microbial 


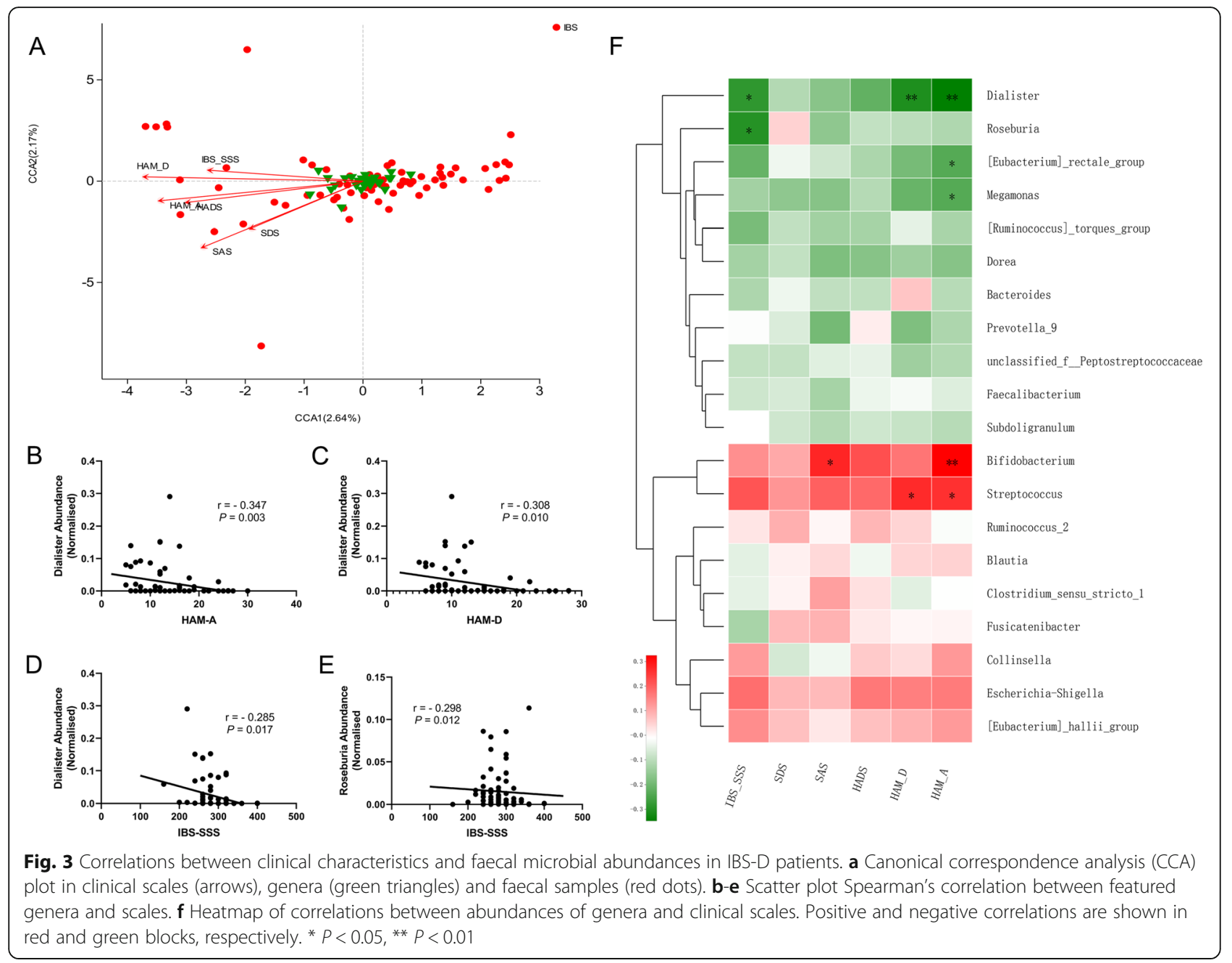

structure. In the adjusted multivariate model, we detected significant negative associations between Dialister quantity with SAS and SDS as well as Clostridium sensu stricto with HADS and HAM-D. The relative abundance of Bifidobacterium was positively associated with anxiety scores in HAM-A and SAS, in parallel with Spearman's correlation results (Table S2).

\section{Differentiated urinary metabolic profiles of IBS-D patients and $\mathrm{HCs}$}

Ultra-high performance liquid chromatography tandem mass spectrometry (UHPLC-MS/MS) harvested urinary metabolomic data from 70 IBS-D and $42 \mathrm{HC}$ samples. Orthogonal partial least squares-discrimination analysis (OPLS-DA) was employed to explore the differentiated metabolites between two groups (Figs. 4a, b). The robustness of OPLS-DA models was verified by 7 -fold crossvalidation $\left(\mathrm{Q}^{2}=0.544\right.$ for $\mathrm{ESI}^{-}$mode and $\mathrm{Q}^{2}=0.511$ for $\mathrm{ESI}^{+}$mode) and 200 times of permutation tests were utilised to exclude overfitting models $\left(\mathrm{Q}^{2}\right.$ intercept $<0$ for both $\mathrm{ESI}^{-}$and $\mathrm{ESI}^{+}$modes). After removal of duplicates, a total of 114 metabolites emerged to be significantly different between IBS-D group and HC group (Variable importance in the projection (VIP) $>1.00$, adjusted $P<0.05$ ), among which 50 metabolites were annotated with Kyoto Encyclopedia of Genes and Genomes (KEGG) compound IDs (Table S3). Differentiated metabolites with the highest VIPs are O-ureido-L-serine (VIP $=6.27$, HC/IBS-D fold change $(\mathrm{FC})=2.23), 3,4$-dihydroxybenzenesulfonic acid $(\mathrm{VIP}=6.24, \quad \mathrm{FC}=2.60), \quad(\mathrm{R})$-2-hydroxyglutarate $\quad(\mathrm{VIP}=$ $5.67, \mathrm{FC}=3.16),\left(2 \mathrm{R} \_3 \mathrm{~S}\right)-2 \_3$-dimethylmalate $(\mathrm{VIP}=5.35$, $F C=0.07)$. After mapping differentiated metabolites into KEGG metabolic pathways and conducting enrichment analysis and topology analysis, caffeine metabolism pathway exhibited highest impact value of 0.31 . However, no metabolism pathways demonstrated significant distinction between IBS-D and HCs (Fig. 4c, Table S4).

\section{Urinary metabolites associated with psychological} parameters and microbiota composition of IBS-D patients In the aim of combining metabolomic outcomes and clinical characteristics of IBS-D patients, we calculated 


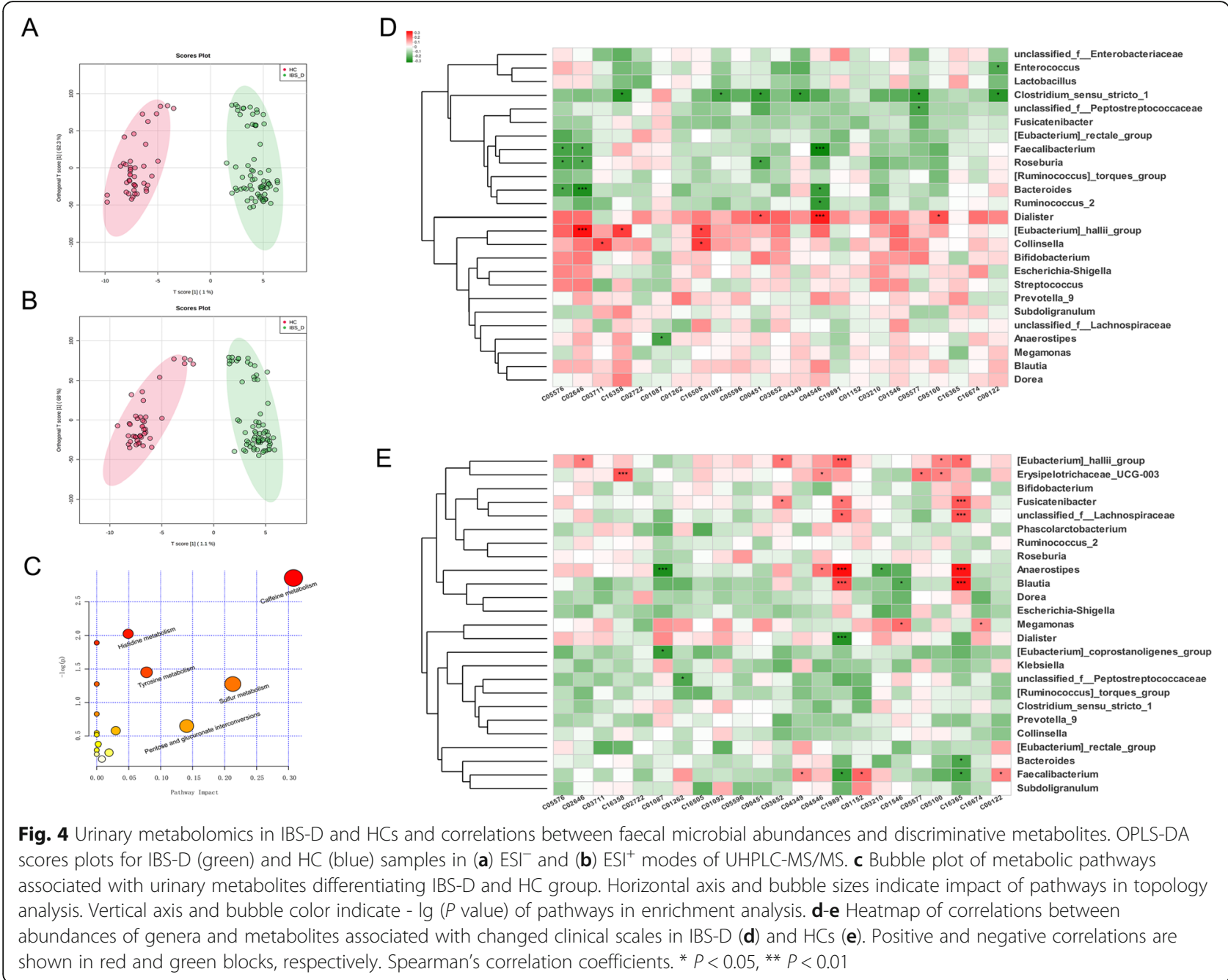

the correlations between differentiated urinary metabolite quantities and IBS clinical parameters (Table 2). Among the metabolites which were negatively associated with depression or anxiety level, 3,4-dihydroxymandelaldehyde manifested best correlation with SAS $(r=-0.383$, $P=0.001$ ), followed by 1 -methylxanthine (with SDS, $r=-0.347, \quad P=0.004), \quad$ 4-hydroxyphenylacetylglycine (with SAS, $\quad r=-0.337, P=0.005$ ) and 1D-chiroinositol (with SAS, $r=-0.336, P=0.005$ ). Some metabolites also manifested positive or negative correlations with IBS symptom severity, including 2-furoate $(r=0.424, \quad P<0.001)$ and anserine $(r=-0.296, \quad P=$ 0.002), etc. Interestingly, 1-methylhistidine showed negative associations with not only symptom severity $(r=-0.260, P=0.007)$ but SAS $(r=-0.263, P=0.007)$ and SDS $(r=-0.250, P=0.031)$. When age, gender and IBS symptom severity were adjusted for, significant relationships between urinary quantities of annotated metabolites and psychological outcomes were shown in the multivariate linear regression models (Table S5).
To further investigate the relationship between urinary metabolites and faecal microbiome, correlation matrices based on spearman's coefficient were constructed for IBS-D and HC group, respectively (Fig. 4d, e). For IBS-D patients, both 3,4-dihydroxymandelaldehyde and 1methylxanthine were negatively correlated with the relative abundance of Clostridium sensu stricto. Significant relationships were also found in positive correlation between (R)-3-((R)-3-hydroxybutanoyloxy) butanoate and Dialister $(r=0.312, P=0.008)$, and negative association of (R)-3-((R)-3-hydroxybutanoyloxy) butanoate and Faecalibacterium $(r=-0.335, P=0.005)$, etc. However, none of these significant relationships was demonstrated in the correlation matrix of $\mathrm{HC}$ population, suggesting the distinction of microbe-related metabolites between two groups. Quantitative data of metabolites and gut microbial genera with abundances $>1 \%$ were also used to construct correlation networks to visualize a full-scale view of the microbiome-metabolite relationship via Cytoscape platform (Fig. S2). 
Table 2 Associations between clinical characteristics and differential urinary metabolites in IBS-D patients

\begin{tabular}{|c|c|c|c|c|}
\hline KEGG ID & Metabolite Name & Related Parameter & Spearman's Coefficient & $P$ value \\
\hline C05576 & 3_4-Dihydroxyphenylethyleneglycol & ${\mathrm{IBS}-S S S^{\mathrm{a}}}$ & 0.216 & 0.026 \\
\hline C02722 & n-Hexanamide & IBS-SSS & 0.208 & 0.032 \\
\hline C01546 & 2-Furoate & IBS-SSS & 0.424 & 0.000 \\
\hline C16505 & (10S)-Juvenile hormone III diol & IBS-SSS & 0.366 & 0.000 \\
\hline C16674 & formyl-isoglutamine & IBS-SSS & 0.329 & 0.001 \\
\hline C01262 & Anserine & IBS-SSS & -0.296 & 0.002 \\
\hline C01152 & 1-Methylhistidine & IBS-SSS & -0.260 & 0.007 \\
\hline C03652 & (2R_3S)-2_3-Dimethylmalate & IBS-SSS & 0.250 & 0.009 \\
\hline C16365 & 5-Acetylamino-6-formylamino-3-methyluracil & IBS-SSS & -0.236 & 0.014 \\
\hline C19891 & 1D-chiro-inositol & IBS-SSS & -0.230 & 0.017 \\
\hline C00122 & Fumaricacid & IBS-SSS & -0.218 & 0.024 \\
\hline C00451 & (1R_2S)-1-Hydroxypropane-1_2_3-tricarboxylate & IBS-SSS & 0.196 & 0.043 \\
\hline C03210 & 2_2'-Iminodipropanoate & IBS-SSS & 0.192 & 0.047 \\
\hline C16358 & 1-Methylxanthine & $S A S^{b}$ & -0.286 & 0.019 \\
\hline C05577 & 3_4-Dihydroxymandelaldehyde & SAS & -0.383 & 0.001 \\
\hline C05596 & 4-Hydroxyphenylacetylglycine & SAS & -0.337 & 0.005 \\
\hline C19891 & 1D-chiro-inositol & SAS & -0.336 & 0.005 \\
\hline C16674 & formyl-isoglutamine & SAS & -0.306 & 0.012 \\
\hline C04349 & (4S)-4_6-Dihydroxy-2_5-dioxohexanoate & SAS & -0.294 & 0.016 \\
\hline C00122 & Fumaricacid & SAS & -0.292 & 0.017 \\
\hline C05100 & 3-Ureidoisobutyrate & SAS & -0.281 & 0.021 \\
\hline C01152 & 1-Methylhistidine & SAS & -0.263 & 0.031 \\
\hline C16358 & 1-Methylxanthine & $\operatorname{SDS}^{c}$ & -0.347 & 0.004 \\
\hline C03711 & N-Methylphenylethanolamine & SDS & -0.266 & 0.030 \\
\hline C02646 & 4-Coumarylalcohol & SDS & -0.245 & 0.046 \\
\hline C03652 & (2R_3S)-2_3-Dimethylmalate & SDS & -0.268 & 0.028 \\
\hline C05577 & 3_4-Dihydroxymandelaldehyde & SDS & -0.267 & 0.029 \\
\hline C01087 & (R)-2-Hydroxyglutarate & SDS & -0.266 & 0.030 \\
\hline C01092 & 8-Amino-7-oxononanoate & SDS & -0.265 & 0.030 \\
\hline C01152 & 1-Methylhistidine & SDS & -0.250 & 0.042 \\
\hline C05596 & 4-Hydroxyphenylacetylglycine & SDS & -0.242 & 0.049 \\
\hline C04546 & (R)-3-((R)-3-Hydroxybutanoyloxy)butanoate & SDS & -0.242 & 0.049 \\
\hline C01262 & Anserine & HAM-A ${ }^{d}$ & -0.253 & 0.039 \\
\hline
\end{tabular}

IBS-SSS IBS symptom severity scale, ${ }^{\mathrm{b}}$ SAS Self-reported anxiety scale, ${ }^{\mathrm{C}}$ SDS Self-reported depression scale, ${ }^{\mathrm{d}} H A M-A$ Hamilton anxiety scale

\section{Discussion}

In this study, we examined the relationship between clinical and psychological abnormalities, faecal microbiome and urinary metabolic profile in IBS-D patients, potentially supporting further mechanism researches. To our knowledge, this is the first study to combine faecal microbiome and urinary metabolome data with psychological symptoms in IBS to date.

Previous studies have determined that IBS patients featured gut microbiota alterations, which contributed to IBS pathophysiology [16]. However, results concerning microbial diversity and composition were divergent, which might arise from the heterogeneity of IBS. Our current study compared the faecal microbiota from IBS$\mathrm{D}$ and healthy groups. Simpson index demonstrated decreased faecal biodiversity, whereas Ace and Chao indices showed higher microbial richness in IBS-D group, supported by human and animal researches [17-19]. Analysis of similarity (ANOSIM), PCoA and hierarchical clustering analysis based on Bray-Curtis distance showed no significant difference of $\beta$-diversity between groups. Inconsistently, some studies conducted in Chinese or 
western population also revealed decreased or unchanged microbial $\alpha$-diversity and richness in IBS-D [8, $18,20,21]$. Interestingly, "normal-like" microbiota in IBS subgroup, which might result in undetected selection bias and inconsistency of overall compositional results, was suggested by accumulating reports [22, 23]. From the perspective of bacterial taxonomic alterations, most high-throughput data showed a decreased abundance of phyla Firmicutes and increased Bacteroidetes in IBS microbes in agreement with our results [21, 24]. Vast studies demonstrated that Bifidobacterium and Lactobacillus were less abundant in IBS-D, whereas Clostridium, Escherichia coli and Enterobacter were often increased [19, 20, 24-26]. Faecalibacterium (or F. prausnitzii) abundance in IBS increased in some studies [27], but constant or decreased in others [24]. However, a recent meta-analysis that included 17 studies argued that species-specific alterations of gut microbiota were different between IBS patients from China and other regions [25]. Differentiation of microbial composition also existed among different IBS sub-categories (especially between IBS-D and constipation-predominant IBS), probably due to distinct manifestations of IBS bowel motility [24, 27, 28]. Here we found Faecalibacterium, Subdoligranulum and Eubacterium rectale group, all capable of producing butyrate [29], were depleted in IBS-D faecal samples. Researches have demonstrated that short chain fatty acids (SCFAs), of which butyrate is more extensively studied [30], are intestinal fermentative products that play a critical role in protecting intestinal mucosa, regulating innate and systematic immune response and improving gastrointestinal motility [31]. For IBS patients, SCFA-producing microorganisms such as Roseburia, Faecalibacterium, Eubacterium and Ruminococcaceae family and were reported depleted [8, 32-34]. Interestingly, low-FODMAP diet, which effectively improved IBS bloating and distension symptoms [13, 35], could also result in reduced intestinal butyrate abundance and butyrate-producing bacteria [13, 36-38]. These findings suggested the complex interplay between diets, IBS microbiome and symptoms, and warranted further dietmanagement investigations. It's also interesting that Prevotella abundance increased in IBS-D group. Previous studies have shown that Prevotella was associated with immunological imbalance and localised or systemic diseases, probably through promoting mucosal Th17 immune responses [39-42]. A recent report revealed that Prevotella was depleted in faeces from IBS patients and post-infectious IBS (PI-IBS) mice model, and might be positively associated with a high risk of development of IBS hypersensitivity [20]. Considering the theory that IBS embodies low-grade and chronic inflammation [43], further investigations on the role of Prevotella in IBS and other gut inflammatory alterations are of interest.
Metabolomics has been broadly acknowledged to be closely associated with phenotypes in health and diseases [44]. Earlier targeted metabolomics studies primarily focused on several categories of metabolites, including SCFAs, amino acids, bile acids, etc. [26, 45]. In contrast, non-targeted metabolomics approaches under development collected more detailed and global metabolic profiling data concerning metabolic homeostasis and aberrance [14, 19, 46, 47]. Growing data had demonstrated that metabolism was disturbed in IBS of animal model [48] or patients [14], whereas detected metabolites were in divergence among studies. Faecal or serum samples used to be largely utilised in metabolomic studies of IBS and other diseases. In comparison, urine was much easier to obtain at an outpatient clinic, considered sterile, and free from bio-macromolecules [15], therefore could be apt for metabolomic analysis. Recent research on proton nuclear magnetic resonance $\left({ }^{1} \mathrm{HNMR}\right)$-based urinary metabolomics recruited 16 healthy volunteers and 8 IBS-D patients and indicated lower concentration of indoxyl-sulphate and increase of methylamine and taurine in IBS-D [49]. Another study recruited 76 IBS participants at different time points and detected 10 metabolites consistently elevated in IBS, including a series of hydroxylysine metabolites, amino acids and SCFA derivatives [50]. Here our data harvested 114 differentiated metabolites (50 with KEGG ID annotation) between IBS-D patients and HCs, demonstrating higher sensitivity of UHPLC-MS/MS-based metabolomics (relevant studies listed in Table S6). Among the annotated metabolites, some organic acids (3,4-dihydroxybenzenesulfonic acid, (R)-2-hydroxyglutarate, 2,3-dimethylmalate, (1R, 2S)-1-hydroxypropane-1,2,3-tricarboxylate, etc.) and Nphenylacetylglutamine featured highest VIPs telling IBSD from HC samples. However, no statistically significant difference was observed in the pathway enrichment analysis between IBS-D and HCs in our study, in contrast to previous reports that alanine, aspartate, and glutamate metabolism, amino sugar and nucleotide sugar metabolism were disturbed in IBS [14].

Though accumulating researches have discussed microbial and metabolic alterations in IBS, few focused on their relationships with IBS symptoms, especially psychological abnormalities. Herein, we analysed correlations between clinical data and faecal microbial in a comparatively large population of IBS patients. Dialister abundance was negatively correlated with IBS symptom severity, anxiety and depression level. A study of diet therapy demonstrated that more significant improvements in systematic inflammatory levels were related to higher proportions of Dialister [51]. Previous works also suggested that Dialister was deficient in depression (corrected for antidepressant intake) [52] and some paediatric diseases, including food sensitization [53], self- 
immune diseases [54] and autism spectrum disorders [55]. Modest positive associations took places between anxiety status and Bifidobacterium or Streptococcus. Population with chronic stress demonstrated increased gut Streptococcus, which could be attenuated by probiotic Lactobacillus gasseri. Inconsistently with our results, probiotic preparations of Bifidobacterium improved mental state in previous researches $[12,56]$. We also found that relative abundance of Roseburia was negatively correlated with IBS severity. Roseburia produces SCFAs, mainly butyrate, in the human colon [57]. Studies have reported that patients with constipatedpredominated IBS [32] and ulcerative colitis also demonstrated reduced Roseburia [58]. Treatment of $R$. hominis seemed to be anti-inflammatory in an immuneregulating manner [58-60]. Our previous study [61] has shown the effectiveness of Clostridium butyricum, which is also a butyrate producer in genus Clostridium sensu stricto, in improving IBS-D symptoms and a similar potential of Roseburia in IBS may be worth further investigation. Considering the potential heterogeneity of microbiota driven by age, sex [62] and symptom severity [63], we also built multivariate linear regression models with adjustment of those parameters. Interestingly, in addition to previously detected genera, Clostridium sensu stricto was negatively associated with depression level in the final model.

Moreover, in analysing correlations between clinical data and differentiated urinary metabolites, some metabolites were found positively or negatively associated with IBS severity, anxiety or depression level. A large proportion of those metabolites mainly involved in amino acid metabolism, including tyrosine metabolism (3,4-dihydroxymandelaldehyde, 4-hydroxyphenylacetylglycine), histidine metabolism (1-methylhistidine, anserine) and beta-alanine metabolism (anserine). Alterations in amino acid metabolism were also reported in IBD, celiac disease and intestinal malignancies [64-67]. In our results, the anxiety level of IBS-D patients was negatively correlated with urinary 1D-chiro-inositol, an enantiomer of myo-inositol. Concentration of myo-inositol in central nervous system had been reported to be altered in autism [68, 69] and depression of adolescents [70]. Additionally, magnetic resonance spectroscopy - derived brain myo-inositol concentration could be positively predicted by both intestinal Bacteroides and Clostridium $X I V b$, another suggestion that microbe affects mental status via metabolic regulation [71]. Negative relationships existed between 1-methylxanthine and SDS as well as between 3,4-dihydroxymandelaldehyde and SAS. It was reported that caffeine helped attenuate depression and anxiety behaviour in a dose-dependent manner [72], and it was not surprising that its metabolite, 1methylxanthine, was a negative indicator for depression scale. IBS-D patients had a reduced 3,4-dihydroxymandelaldehyde level in urine observed in our data. In vivo, it was a monoamine oxidase aldehyde metabolite of norepinephrine. Previous research suggested that increased urinary excretion of norepinephrine metabolites (including 3,4-dihydroxymandelaldehyde and vanillylmandelic acid) was predictive of improvement in depressive and anxiety states [73].

Our study combined clinical, microbial and metabolomic data and suggested a possible correlation network among them, as previous evidence suggested the function of microbiota in regulating emotions was potentially in a metabolism modifying manner $[74,75]$. For instance, the anxiety-like phenotype was induced in recipient mice by transplantation of IBS-D faeces, in parallel with changed serum metabolism profiles [75]. In the current study, correlation analysis hinted that two mental state-related metabolites, 1-methylxanthine and 3,4dihydroxymandelaldehyde, were in negative correlation with the relative abundance of Clostridium sensu stricto, suggesting this genus might relate with worsening of psychological abnormality. Social stressor induced an increased abundance of Clostridium in mice [76], and an elevated Clostridium and butyrate in faeces were seen in IBS patients [77]. Clostridium-produced butyrate, though commonly accepted as an anti-inflammatory and protective factor in IBS, may also promote intestinal hypersensitivity seen in animal and human $[61,78,79]$. The potential mechanisms of butyrate aggravated bowel hypersensitivity were reported to involve peptidergic Cfibres, spinal cord plasticity, acid-sensing ion channels 1A (ASIC1A) and modulation of 5-hydroxytryptamine (5-HT or serotonin) release [80]. Also, isobutyrate, another metabolite of Clostridium, was found correlated significantly with decreased anxiolytic and antidepressive effects of prebiotics [81]. In our previous study, Clostridium sensu stricto was depleted in IBS patients with improved quality of life after interfering with probiotics, suggesting its role of predicting posttreatment outcome [61]. Positive correlation between (R)-3-((R)-3-hydroxybutanoyloxy) butanoate and Dialister was also in alignment with the finding that Dialister indicated a lower depression level. However, organisms related to metabolism disturbances shown in correlation matrix were not in proper alignment with those differed between HCs and IBS-D patients, and the specific metabolism-regulating mechanisms of intestinal flora impacting on IBS emotions and symptoms warrant further in vivo researches.

We acknowledge that there are several limitations in our study. Firstly, the collected microbiota, metabolomic data and clinical metadata lacked dietary information and follow-up investigation, potentially confounding the present results. Secondly, the sample size of our single- 
centre exploratory study was still small, which limited the generalization of the conclusions. However, the present sample size had a sufficient statistical power for comparing microbiome between IBS-D and HC groups (see in Methods). Selection bias of study subjects might exist, though main confounding factors including gender and age were controlled in the multivariate linear regression analyses. It's also emphasized that further mechanism studies are required to develop clinical tools concerning microbe- or metabolism-regulated mental health especially in IBS.

\section{Conclusion}

In the current study, we identified specific urinary metabolites and faecal microbes which had the potential to be biomarkers for differentiating IBS patients and healthy controls, though inconsistency with former studies and limited sample size warranted further validation. We also demonstrated that specific microbial genera and metabolites were correlated with not only IBS bowel symptoms but also psychological status including depression and anxiety, suggesting microbiome- or metabolism-oriented diagnostic tools or treatments in IBS would be worth further investigation. Modest to strong associations were observed between metabolites and microbes, corroborating the theory that gut microbes were likely to interplay with physical and mental status via interfering with human metabolism.

\section{Methods}

\section{Participants}

IBS-D patients fulfilling Rome IV criteria were recruited from Department of Gastroenterology at Qilu Hospital of Shandong University between September 2016 and May 2018. The aim of enrolling only IBS-D patients was to eliminate the potential difference between IBS subphenotypes and focus on the most prevalent one in China. Patients suspected of PI-IBS were also excluded due to the heterogeneity in pathophysiology and probable bias in microbial composition. Meanwhile, HC subjects were recruited through advertisement and were defined as individuals with colonoscopy of no negative results and without current or previous gastroenterological symptoms nor history of chronic diseases. None of enrolled subjects were on medication including antibiotics, probiotics, prebiotics, laxatives, non-steroidal antiinflammatory drugs, proton pump inhibitors, steroids, mast cell stabilizers, histamine antagonists, immunosuppressant agents and depressants. No patients nor control subjects were diagnosed with organic diseases including inflammatory bowel disease, coeliac disease, anaphylactic diseases and clinical psychiatric disorders. No patients nor control subjects had undergone gallbladder surgery ever or other major surgeries within half a year.
Basic information and IBS-SSS [82] and Bristol stool scale were provided by participants of both groups. SAS, SDS [83], HADS [84] and HAM-A, HAM-D [85] were utilised to measure anxiety and depression level of IBSD patients by experienced physicians.

\section{Sample collection and storage}

Stool samples and urine samples were collected from IBS-D patients and HCs and kept in empty sterile tubes. All the stool and urine samples were stored at $-80^{\circ} \mathrm{C}$ as soon as possible (delay in transfer less than $5 \mathrm{~min}$ ). Minimal mass required for stool sample per tube was $1.0 \mathrm{~g}$ and minimal volume for urine sample per tube was 5.0 $\mathrm{ml}$. All samples were collectively transferred to Majorbio (Shanghai, China) for further analysis.

\section{$16 \mathrm{~S}$ ribosomal RNA (rRNA) gene sequencing}

Microbial DNA was extracted from fecal samples from IBS-D patients and HCs using the E.Z.N.A. ${ }^{\circ}$ Soil DNA Kit (Omega Bio-tek) according to kit protocols. DNA concentration and purity were assessed by Nanodrop (Thermo Scientific) and DNA quality was determined by $1 \%$ agarose gel electrophoresis. The V3-V4 regions of the bacteria 16S rRNA gene were amplified by PCR using the primers: 338F 5' -ACTCCTACGGGAGGCAGCA-3' and 806R 5' GGACTACHVGGGTWTCTAAT-3'. The PCR products were then extracted from $2 \%$ agarose gels and further purified by using the AxyPrep DNA Gel Extraction Kit (Axygen Biosciences) and quantified by QuantiFluor ${ }^{\mathrm{Tw}}-\mathrm{ST}$ (Promega) according to protocols. The purified DNA amplicons were then conducted by Majorbio (Shanghai, China) and were sequenced on Illumina MiSeq platform according to the standard protocols.

Harvested reads were stored and mostly analysed on Majorbio i-Sanger Cloud Platform (http://www.i-sanger. com). Reads were firstly trimmed and clustering of similar sequences into OTU was processed with a $97 \%$ sequence identity using Usearch (version $7.0 \mathrm{http} / / /$ drive 5 . com/uparse/). Dereplication, discard of singletons (no less than 5 reads in at least 5 samples) and rarefaction based on the minimal number of reads among samples were conducted before analysis. Ace index and Chao index were calculated to evaluate microbial richness in each sample, and Shannon index, Simpson index for alpha-diversity. Sequences from all samples were classified by Bayesian algorithm using the Ribosomal Database Project (RDP) classifier. Hierarchical clustering and PCoA on a level of all OTUs were performed based on bray-curtis distances. ANOSIM based on Bray-Curtis distance and 999 times of permutation tests was utilised to analyse structural difference between IBS-D and HC groups via R. LEfSe calculation was conducted from phylum to genus level between two groups at the LDA score threshold of 2.5 . 


\section{UHPLC-MS/MS}

Metabolism analysis was performed using a Ultimate 3000 ultra-high performance liquid chromatography system (Dionex, Sunnyvale, CA, United States) united with Q Exactive $^{\mathrm{TM}}$ Hybrid Quadrupole-Orbitrap Mass Spectrometer equipped with an electrospray isolation (ESI) source consisting of both positive and negative ion modes (Thermo Fisher Scientific, Waltham, MA, United States). $100 \mu$ l urine per sample was mixed with $100 \mu \mathrm{l}$ pure water containing internal standard, centrifuged at $13000 \mathrm{rpm}, 4{ }^{\circ} \mathrm{C}$ for $10 \mathrm{~min} .5 \mu \mathrm{l}$ of supernatant per sample was transferred to vial for UHPLC-MS/MS analysis. LC conditions were as follows: column, Acquity UPLC HSS T3 column $(100 \mathrm{~mm} \times 2.1 \mathrm{~mm}, 1.7 \mu \mathrm{m}$, Waters, USA); temperature, $40{ }^{\circ} \mathrm{C}$; flow rate, $0.35 \mathrm{ml} / \mathrm{min}$; separation was achieved by using the following gradient: 0$1.0 \mathrm{~min}, 5 \% \mathrm{~B} ; 1.0-9.0 \mathrm{~min}, 5 \sim 100 \% \mathrm{~B} ; 9.0-12.0 \mathrm{~min}$, $1000 \% \mathrm{~B}, 12.0-15.0 \mathrm{~min}, 5 \% \mathrm{~B}$, where $\mathrm{B}$ is acetonitrile $(0.1 \%(\mathrm{v} / \mathrm{v})$ formic acid) and $\mathrm{A}$ is aqueous formic acid $\left(0.1 \%(\mathrm{v} / \mathrm{v})\right.$ formic acid). The $\mathrm{Q}$ Exactive $^{\mathrm{TM}}$ mass spectrometer was used for its ability to acquire MS/MS spectra on information-dependent acquisition (IDA) mode in the control of the acquisition software (Xcalibur 4.0.27, Thermo). In this mode, the acquisition software continuously evaluates the full scan MS spectrum. The ESI source conditions were set as following: sheath gas flow rate as $45 \mathrm{Arb}$, Aux gas flow rate as 15Arb, capillary temperature $400{ }^{\circ} \mathrm{C}$, full $\mathrm{MS}$ resolution as 70 , $000, \mathrm{MS} / \mathrm{MS}$ resolution as 17,500 , collision energy as $20 / 40 / 60 \mathrm{eV}$ in NCE mode, spray Voltage as $4.0 \mathrm{kV}$ (positive) or $-3.6 \mathrm{kV}$ (negative), respectively. The raw data were converted to the mzML format using ProteoWizard and processed with an in-house program, which was developed using $\mathrm{R}$ and based on XCMS, for peak detection, extraction, alignment, and integration. Then an in-house MS2 database (BiozeronDB) was applied in metabolite annotation. The cut off value for annotation was set at 0.3 . To guarantee the reliability of metabolomic results, quality control (QC) samples were obtained as mixture of aliquots from all urinary samples, and were evenly distributed at intervals of every 10 tested samples throughout the analytical array. Extracted data raw data were trimmed using Compound Discoverer 2.1 (Thermo Fisher Scientific, Waltham, MA, United States) and imported into SIMCA (version 14.0, Umetrics, Sweden) for PCA and OPLS-DA. Comparisons of metabolite concentrations between IBS-D and HC groups were performed using student's t-test. Differentiated metabolites were harvested with criteria of adjusted $P<0.05$, VIP $>1.0$ and then annotated by KEGG pathway database. Enrichment analysis and topological analysis were performed on MetaboAnalyst platform (https://www.metaboanalyst.ca/MetaboAnalyst/faces/ ModuleView.xhtml).

\section{Statistical analyses}

Pearson's Chi-squared test was used in proportion data and Welch's t-test in data with normal distribution. For microbiota analysis, Welch's t-test was used in diversity index calculation. Different taxa between two groups was determined by Mann-Whitney U test. CCA was utilised to analyse the relationship between gut microbial structure and clinical metadata. Redundant variables were gradually removed until all of included variables featured a variance inflation factor below 10. In CCA model, contribution of each environmental factor (clinical metadata) to microbial structure modification was calculated based on 999 Monte Carlo permutations followed by Benjamini-Hochberg correction using envfit function of $\mathrm{R}$. We used multivariate linear regression models to assess the association between psychological scale parameters and microbial abundances/metabolite quantities. We tested the 20 most abundant genera or 50 KEGG ID-annotated metabolites in IBS-D as potential covariances. We built independent models for each genus with age, gender, and IBS severity (IBS-SSS $<300$ as mild, $\geq 300$ as severe) forced in. Genera with a $P$ value under 0.05 were selected into the final multivariate linear regression models. Spearman correlation coefficients were calculated for relationships between environmental factors, taxonomic abundances and metabolomic data. Correlation network between metabolomic and microbial data was plotted (correlation edges with spearman's coefficient $>0.3 /<-0.3$ and $P<0.05$ were shown) and visualised by the open-source Cytoscape v.3.2.2. We conducted post hoc power calculation for the comparison of differential OTU abundances between IBS and HC groups (stricter than simply compare alphaand beta-diversity) using a published Shiny-based microbiome power calculator (http://fedematt.shinyapps.io/ shinyMB/) [86]. The Monte Carlo simulation and Wilcoxon-Mann-Whitney test based calculator demonstrated that our current samples (70 IBS and 44 HC) had sufficient power (0.81) to detect a $30 \%$ fluctuation in at least 4 most abundant OTUs between 2 groups. All $P$ values were two-sided. FDR following BenjaminiHochberg procedure was applied under multiple-testing circumstances. $P$ or adjusted $P$ values below 0.05 were considered statistically significant. Statistical analyses were performed using SPSS version 23.0 (SPSS, Inc., Chicago, IL, United States) and R v.3.6.2.

\section{Supplementary information}

Supplementary information accompanies this paper at https://doi.org/10. 1186/s12866-020-01841-4.

Additional file 1.

Additional file 2.

Additional file 3. 


\section{Additional file 4.}

Additional file 5.

\section{Abbreviations}

IBS: Irritable bowel syndrome; low-FODMAP: Low Fermentable oligo-dimono-saccharides and polyols; IBS-D: Diarrhoea-predominant irritable bowel syndrome; BMI: Body mass index; HC: Healthy control; IBS-SSS: IBS symptom severity scale; PCOA: Principal co-ordinates analysis; OTU: Operational taxonomy unit; FDR: False discovery rate; LEfSe: Linear discriminant analysis effect size; LDA: Linear discriminant analysis; SAS / SDS: Self-reported Anxiety and Depression Scales; HADS: Hospital Anxiety and Depression Scale; HAM-A / HAM-D: Hamilton Anxiety and Depression Scales; CCA: Canonical correspondence analysis; UHPLC MS/MS: Ultra-high performance liquid chromatography tandem mass spectrometry; OPLS-DA: Orthogonal partial least squares-discrimination analysis; VIP: Variable importance in the projection; KEGG: Kyoto Encyclopedia of Genes and Genomes; FC: Fold change; ANOSIM: Analysis of similarity; SCFAs: Short chain fatty acids; PlIBS: Post-infectious irritable bowel syndrome; 1HNMR: Proton nuclear magnetic resonance

\section{Acknowledgements}

We deeply appreciate Lei-Qi Xu, Yi-Yuan Sun, Lin Lin, Tong-Yu Wang, JunYan Qu, Chao-Ran Ji, Jing Liu, Kai-Ruo Wang, Ru-Chen Zhou and Pei-Zhu Wang for their valuable supports and advices during the research and manuscript drafting.

\section{Authors' contributions}

XLZ initiated and supervised the study. TL, $L X L$ and XLZ designed the study and were major contributors in writing and revising the manuscript. TL collected the samples. TL, ML, LXL and XG were major contributors in conducting statistical analysis and interpretation of data. $\mathrm{XG}, \mathrm{XC}$ and $\mathrm{BL}$ provided technical assistance. All authors have read and approved the manuscript.

\section{Funding}

This study was funded by the National Natural Science Foundation of China (81770538 and 81570485), Key Research and Development Program of Shandong Province (2017CXGC1215). Funding bodies had no role in study design, data collection, analysis, and writing manuscript.

\section{Availability of data and materials}

The dataset of 165 rRNA gene sequencing supporting the conclusions of this article is available in the National Center Biotechnology Information repository with accession code PRJNA544721. Urinary metabolomic raw data has been deposited to Figshare repository with the assigned DOI: https://doi. org/10.6084/m9.figshare.8218889.

\section{Ethics approval and consent to participate}

The study was performed in accordance of Declaration of Helsinki and approved by the ethics committee in Qilu Hospital of Shandong University. All of subjects gave written informed consent prior to recruitment and sample collection.

\section{Consent for publication}

Not applicable.

\section{Competing interests}

The authors declare that they have no competing interests.

\section{Author details}

'Department of Gastroenterology, Qilu Hospital, Cheloo College of Medicine, Shandong University, 107 Wenhuaxi Road, Jinan 250012, Shandong Province, China. ${ }^{2}$ Laboratory of Translational Gastroenterology, Qilu Hospital, Cheloo College of Medicine, Shandong University, Jinan, China. ${ }^{3}$ Robot engineering laboratory for precise diagnosis and therapy of Gl tumour, Qilu Hospital, Cheloo College of Medicine, Shandong University, Jinan, China.
Received: 19 January 2020 Accepted: 3 June 2020

Published online: 17 June 2020

\section{References}

1. Sperber AD, Dumitrascu D, Fukudo S, Gerson C, Ghoshal UC, Gwee KA, et al. The global prevalence of IBS in adults remains elusive due to the heterogeneity of studies: a Rome Foundation working team literature review. Gut. 2017;66:1075-82..

2. Lovell RM, Ford AC. Global prevalence of and risk factors for irritable bowel syndrome: a meta-analysis. Clin Gastroenterol Hepatol. 2012;10:712-721.e4. https://doi.org/10.1016/j.cgh.2012.02.029.

3. Drossman DA, Hasler WL. Rome IV-functional GI disorders: disorders of gutbrain interaction. Gastroenterology. 2016;150:1257-61.

4. Koloski NA, Jones M, Kalantar J, Weltman M, Zaguirre J, Talley NJ. The brain gut pathway in functional gastrointestinal disorders is bidirectional: a 12year prospective population-based study. Gut. 2012;61:1284-90.

5. Whitehead WE, Palsson O, Jones KR. Systematic review of the comorbidity of irritable bowel syndrome with other disorders: what are the causes and implications? Gastroenterology. 2002;122:1140-56.

6. Barbara G, Feinle-Bisset C, Ghoshal UC, Santos J, Vanner SJ, Vergnolle N, et al. The intestinal microenvironment and functional gastrointestinal disorders. Gastroenterology. 2016;150:1305-1318.e8.

7. Carroll IM, Tamar RK, Keku TO, Young-Hyo C, Packey CD, Balfour RS, et al. Molecular analysis of the luminal- and mucosal-associated intestinal microbiota in diarrhea-predominant irritable bowel syndrome. Am J Physiol Gastrointest Liver Physiol. 2011;301:799-807.

8. Carroll IM, Ringel-Kulka T, Siddle JP, Ringel Y. Alterations in composition and diversity of the intestinal microbiota in patients with diarrhea-predominant irritable bowel syndrome. Neurogastroenterol Motil. 2012;24:521-e248.

9. Zheng P, Zeng B, Zhou C, Liu M, Fang Z, Xu X, et al. Gut microbiome remodeling induces depressive-like behaviors through a pathway mediated by the host's metabolism. Mol Psychiatry. 2016;21:1-11. https://doi.org/10. 1038/mp.2016.44.

10. De Palma G, Lynch MDJ, Lu J, Dang VT, Deng Y, Jury J, et al. Transplantation of fecal microbiota from patients with irritable bowel syndrome alters gut function and behavior in recipient mice. Sci Transl Med. 2017;9:1-15.

11. Shankar V, Reo NV, Paliy O. Simultaneous fecal microbial and metabolite profiling enables accurate classification of pediatric irritable bowel syndrome. Microbiome. 2015;3:73.

12. Pinto-Sanchez MI, Hall GB, Ghajar K, Nardelli A, Bolino C, Lau JT, et al. Probiotic Bifidobacterium longum NCC3001 reduces depression scores and alters brain activity: a pilot study in patients with irritable bowel syndrome. Gastroenterology. 2017;153:448.

13. McIntosh K, Reed DE, Schneider T, Dang F, Keshteli AH, De Palma G, et al. FODMAPs alter symptoms and the metabolome of patients with IBS: a randomised controlled trial. Gut. 2017;66:1241-51.

14. Zeber-Lubecka N, Kulecka M, Ambrozkiewicz F, Paziewska A, Goryca K, Karczmarski J, et al. Limited prolonged effects of rifaximin treatment on irritable bowel syndrome-related differences in the fecal microbiome and metabolome. Gut Microbes. 2016;7:397-413.

15. Bouatra S, Aziat F, Mandal R, Guo AC, Wilson MR, Knox C, et al. The human urine metabolome. PLoS One. 2013;8:e73076.

16. Chey W, Menees S. The gut microbiome and irritable bowel syndrome. F1000Res. 2018:7:1-10

17. Smith PA. The tantalizing links between gut microbes and the brain. Nature. 2015. https://doi.org/10.1038/526312a.

18. Stilling RM, van de Wouw M, Clarke G, Stanton C, Dinan TG, Cryan JF. The neuropharmacology of butyrate: the bread and butter of the microbiotagut-brain axis? Neurochem Int. 2016. https://doi.org/10.1016/j.neuint.2016. 06.011.

19. Zhu S, Liu S, Li H, Zhang Z, Zhang Q, Chen L, et al. Identification of gut microbiota and metabolites signature in patients with irritable bowel syndrome. Front Cell Infect Microbiol. 2019;9:1-12.

20. Su T, Liu R, Lee A, Long Y, Du L, Lai S, et al. Altered intestinal microbiota with increased abundance of prevotella is associated with high risk of diarrhea-predominant irritable bowel syndrome. Gastroenterol Res Pract. 2018:2018:11-3.

21. Zhuang X, Tian Z, Li L, Zeng Z, Chen M, Xiong L. Fecal microbiota alterations associated with diarrhea-predominant irritable bowel syndrome. Front Microbiol. 2018;9:1-11. 
22. Jeffery IB, O'Toole PW, Öhman L, Claesson MJ, Deane J, Quigley EMM, et al. An irritable bowel syndrome subtype defined by species-specific alterations in faecal microbiota. Gut. 2012;61:997-1006.

23. Labus JS, Hollister EB, Jacobs J, Kirbach K, Oezguen N, Gupta A, et al. Differences in gut microbial composition correlate with regional brain volumes in irritable bowel syndrome. Microbiome. 2017;5:49.

24. Liu AH, Wu H, Chen Y. Altered molecular signature of intestinal microbiota in irritable bowel syndrome patients compared with healthy controls : a systematic. Dig Liver Dis. 2017. https://doi.org/10.1016/j.dld.2017.01.142.

25. Zhuang $X$, Xiong L, Li L, Li M, Chen M. Alterations of gut microbiota in patients with irritable bowel syndrome: a systematic review and metaanalysis. J Gastroenterol Hepatol. 2016;32:28-38.

26. Duboc H, Rainteau D, Rajca S, Humbert L, Farabos D, Maubert M, et al. Increase in fecal primary bile acids and dysbiosis in patients with diarrheapredominant irritable bowel syndrome. Neurogastroenterol Motil. 2012;24: $1-10$.

27. Sundin J, Aziz I, Nordlander S, Polster A, Hu YOO, Hugerth LW, et al. Evidence of altered mucosa-associated and fecal microbiota composition in patients with irritable bowel syndrome. Sci Rep. 2020;10:1-13.

28. Shukla R, Ghoshal U, Dhole TN, Ghoshal UC. Fecal microbiota in patients with irritable bowel syndrome compared with healthy controls using realtime polymerase chain reaction: an evidence of Dysbiosis. Dig Dis Sci. 2015. https://doi.org/10.1007/s10620-015-3607-y.

29. Venkataraman A, Sieber JR, Schmidt AW, Waldron C, Theis KR, Schmidt TM. Variable responses of human microbiomes to dietary supplementation with resistant starch. Microbiome. 2016;4:33.

30. Ríos-Covián D, Ruas-Madiedo P, Margolles A, Gueimonde M, de Los ReyesGavilán CG, Salazar N. Intestinal short chain fatty acids and their link with diet and human health. Front Microbiol. 2016. https://doi.org/10.3389/fmicb. 2016.00185.

31. Koh A, De Vadder F, Kovatcheva-Datchary P, Bäckhed F. From dietary fiber to host physiology: short-chain fatty acids as key bacterial metabolites. Cell. 2016;165:1332-45.

32. Chassard C, Dapoigny M, Scott KP, Crouzet L, Del'Homme C, Marquet $P$, et al. Functional dysbiosis within the gut microbiota of patients with constipated-irritable bowel syndrome. Aliment Pharmacol Ther. 2012;35: 828-38

33. Pozuelo M, Panda S, Santiago A, Mendez S, Accarino A, Santos J, et al. Reduction of butyrate- and methane-producing microorganisms in patients with irritable bowel syndrome. Sci Rep. 2015:5:12693.

34. Maukonen J, Satokari R, Matto J, Soderlund H, Mattila-Sandholm T, Saarela M. Prevalence and temporal stability of selected clostridial groups in irritable bowel syndrome in relation to predominant faecal bacteria. J Med Microbiol. 2006;55(Pt 5):625-33.

35. Zahedi MJ, Behrouz V, Azimi M. Low fermentable oligo-di-mono-saccharides and polyols diet versus general dietary advice in patients with diarrheapredominant irritable bowel syndrome: a randomized controlled trial. J Gastroenterol Hepatol. 2018;33:1192-9.

36. Hustoft TN, Hausken T, Ystad SO, Valeur J, Brokstad K, Hatlebakk JG, et al. Effects of varying dietary content of fermentable short-chain carbohydrates on symptoms, fecal microenvironment, and cytokine profiles in patients with irritable bowel syndrome. Neurogastroenterol Motil. 2017. https://doi. org/10.1111/nmo.12969.

37. Chen B-R, Du L-J, He H-Q, Kim JJ, Zhao Y, Zhang Y-W, et al. Fructooligosaccharide intensifies visceral hypersensitivity and intestinal inflammation in a stress-induced irritable bowel syndrome mouse model. World J Gastroenterol. 2017. https://doi.org/10.3748/wjg.v23.i47.8321.

38. Halmos EP, Christophersen CT, Bird AR, Shepherd SJ, Muir JG, Gibson PR. Consistent prebiotic effect on gut microbiota with altered fodmap intake in patients with Crohn's disease: a randomised, controlled cross-over trial of well-defined diets. Clin Transl Gastroenterol. 2016. https://doi.org/10.1038/ ctg.2016.22.

39. Ley RE. Gut microbiota in 2015: Prevotella in the gut: choose carefully. Nat Rev Gastroenterol Hepatol. 2016;13:69. https://doi.org/10.1038/nrgastro.2016.4.

40. Pandiyan P, Bhaskaran N, Zou M, Schneider E, Jayaraman S, Huehn J. Microbiome dependent regulation of Tregs and Th17 cells in mucosa. Front Immunol. 2019;10:426.

41. Palareti G, Legnani C, Cosmi B, Antonucci E, Erba N, Poli D, et al. Comparison between different D-dimer cutoff values to assess the individual risk of recurrent venous thromboembolism: analysis of results obtained in the DULCIS study. Int J Lab Hematol. 2016;38:42-9.
42. Scher JU, Sczesnak A, Longman RS, Segata N, Ubeda C, Bielski C, et al. Expansion of intestinal Prevotella copri correlates with enhanced susceptibility to arthritis. Elife. 2013. https://doi.org/10.7554/eLife.01202.

43. Vivinusnébot $M$, Frinmathy $G$, Bzioueche $H$, Dainese $R$, Bernard $G$, Anty $R$, et al. Functional bowel symptoms in quiescent inflammatory bowel diseases: role of epithelial barrier disruption and low-grade inflammation. Gut. 2014;63:744-52

44. Guijas C, Montenegro-Burke JR, Warth B, Spilker ME, Siuzdak G. Metabolomics activity screening for identifying metabolites that modulate phenotype. Nat Biotechnol. 2018;36:316-20.

45. Zhang WX, Zhang Y, Qin G, Li KM, Wei W, Li SY, et al. Altered profiles of fecal metabolites correlate with visceral hypersensitivity and may contribute to symptom severity of diarrhea-predominant irritable bowel syndrome. World J Gastroenterol. 2019;25:6416-29.

46. Lee JS, Kim SY, Chun YS, Chun YJ, Shin SY, Choi CH, et al. Characteristics of fecal metabolic profiles in patients with irritable bowel syndrome with predominant diarrhea investigated using $1 \mathrm{H}-\mathrm{NMR}$ coupled with multivariate statistical analysis. Neurogastroenterol Motil. 2020;3:1-13.

47. Shankar V, Homer D, Rigsbee L, Khamis HJ, Michail S, Raymer M, et al. The networks of human gut microbe-metabolite associations are different between health and irritable bowel syndrome. ISME J. 2015;9:1899-903. https://doi.org/10.1038/ismej.2014.258.

48. Yu LM, Zhao KJ, Wang SS, Wang X, Lu B. Gas chromatography/mass spectrometry based metabolomic study in a murine model of irritable bowel syndrome. World J Gastroenterol. 2018;24:894-904.

49. Noorbakhsh $H$, Yavarmanesh M, Mortazavi SA, Adibi P, Moazzami AA. Metabolomics analysis revealed metabolic changes in patients with diarrhea-predominant irritable bowel syndrome and metabolic responses to a synbiotic yogurt intervention. Eur J Nutr. 2018;58:1-11.

50. Yamamoto $M$, Ines $M$, Sanchez $P$, Bercik $P$, Britz $P$. Metabolomics reveals elevated urinary excretion of collagen degradation and epithelial cell turnover products in irritable bowel syndrome patients. Metabolomics. 2019;15:1-18. https://doi.org/10.1007/s11306-019-1543-0.

51. Inés $\mathrm{M}$, Lattimer JM, Hubach $\mathrm{KL}$, Case JA, Junyi $Y$, Weber $C G$, et al. Gut microbiome composition is linked to whole grain-induced immunological improvements. ISME J. 2013;7:269-80.

52. Valles-Colomer M, Falony G, Darzi Y, Tigchelaar EF, Wang J, Tito RY, et al. The neuroactive potential of the human gut microbiota in quality of life and depression. Nat Microbiol. 2019;4:623-32. https://doi.org/10.1038/ s41564-018-0337-x.

53. Savage JH, Leesarwar KA, Sordillo J, Bunyavanich S, Zhou Y, O'Connor G, et al. A prospective microbiome-wide association study of food sensitization and food allergy in early childhood. Allergy. 2018;73:145.

54. Wang X, Zhang L, Wang Y, Liu X, Zhang H, Liu Y, et al. Gut microbiota dysbiosis is associated with Henoch-Schönlein Purpura in children. Int Immunopharmacol. 2018;58:1-8.

55. Strati F, Cavalieri D, Albanese D, De Felice C, Donati C, Hayek J, et al. New evidences on the altered gut microbiota in autism spectrum disorders. Microbiome. 2017;5:1-11.

56. Nishida K, Sawada D, Kuwano Y, Tanaka H, Rokutan K. Health benefits of lactobacillus gasseri cp2305 tablets in young adults exposed to chronic stress: a randomized, double-blind, placebo-controlled study. Nutrients. 2019:11:1859.

57. Tamanai-Shacoori Z, Smida I, Bousarghin L, Loreal O, Meuric V, Fong SB, et al. Roseburia spp.: a marker of health? Future Microbiol. 2017;12:157-70.

58. Machiels K, Joossens M, Sabino J, De Preter V, Arijs I, Eeckhaut V, et al. A decrease of the butyrate-producing species roseburia hominis and faecalibacterium prausnitzii defines dysbiosis in patients with ulcerative colitis. Gut. 2014;63:1275-83. https://doi.org/10.1136/gutjnl-2013-304833.

59. Aminov RI, Delday MI, Ferraria VC, Garden K, Patterson AM, Grant G, et al. Human gut symbiont roseburia hominis promotes and regulates innate immunity. Front Immunol. 2017;8:1-14.

60. Zhu C, Song K, Shen Z, Quan Y, Tan B, Luo W, et al. Roseburia intestinalis inhibits interleukin 17 excretion and promotes regulatory $T$ cells differentiation in colitis. Mol Med Rep. 2018;17:7567-74.

61. Sun YY, Li M, Li YY, Li LX, Zhai WZ, Wang P, et al. The effect of Clostridium butyricum on symptoms and fecal microbiota in diarrhea-dominant irritable bowel syndrome: a randomized, double-blind, placebo-controlled trial. Sci Rep. 2018;8:1-11. https://doi.org/10.1038/s41598-018-21241-z.

62. Mulak A, Taché $Y$, Larauche M. Sex hormones in the modulation of irritable bowel syndrome. World J Gastroenterol. 2014;20:2433. 
63. Tap J, Derrien M, Törnblom H, Brazeilles R, Cools-Portier S, Doré J, et al. Identification of an intestinal microbiota signature associated with severity of irritable bowel syndrome. Gastroenterology. 2017;152:111-23.

64. Scoville EA, Allaman MM, Brown CT, Motley AK, Horst SN, Williams CS, et al. Alterations in lipid, amino acid, and energy metabolism distinguish Crohn's disease from ulcerative colitis and control subjects by serum metabolomic profiling. Metabolomics. 2018;14. https://doi.org/10.1007/s11306-017-1311-y.

65. Agus A, Planchais J, Sokol H. Gut microbiota regulation of tryptophan metabolism in health and disease. Cell Host Microbe. 2018;23:716-24. https://doi.org/10.1016/j.chom.2018.05.003.

66. Baxter BA, Oppel RC, Ryan EP. Navy beans impact the stool metabolome and metabolic pathways for colon health in cancer survivors. Nutrients. 2019;11:28.

67. Naluai ÅT, Vafa LS, Gudjonsdottir AH, Arnell H, Browaldh L, Nilsson S, et al. Altered peripheral amino acid profile indicate a systemic impact of active celiac disease and a possible role of amino acids in disease pathogenesis. PLoS One. 2018;13:1-8.

68. Friedman SD, Shaw DW, Artru AA, Richards TL, Gardner J, Dawson G, et al. Regional brain chemical alterations in young children with autism spectrum disorder. Neurology. 2003;60:100-7.

69. Gabis L, Wei H, Azizian A, DeVincent C, Tudorica A, Kesner-Baruch Y, et al. $1 \mathrm{H}$-magnetic resonance spectroscopy markers of cognitive and language ability in clinical subtypes of autism spectrum disorders. J Child Neurol. 2008;23:766-74

70. Urrila AS, Hakkarainen A, Castaneda A, Paunio T, Marttunen M, Lundbom N. Frontal cortex Myo-inositol is associated with sleep and depression in adolescents: a proton magnetic resonance spectroscopy study. Neuropsychobiology. 2017;75:21-31.

71. Mudd AT, Berding K, Wang M, Donovan SM, Dilger RN. Serum cortisol mediates the relationship between fecal Ruminococcus and brain Nacetylaspartate in the young pig. Gut Microbes. 2017;8:589-600.

72. Sweeney $P$, Levack R, Watters J, Xu Z, Yang Y. Caffeine increases food intake while reducing anxiety-related behaviors. Appetite. 2016;101:171-7.

73. Schildkraut JJ, Kopin IJ, Schanberg SM, Durell J. Norepinephrine metabolism and psychoactive drugs in the endogenous depressions. 1968. Pharmacopsychiatry. 1968;1:69-92.

74. Soto M, Herzog C, Pacheco JA, Fujisaka S, Bullock K, Clish CB, et al. Gut microbiota modulate neurobehavior through changes in brain insulin sensitivity and metabolism. Mol Psychiatry. 2018;23:2287-301.

75. De Palma G, Lynch MD, Lu J, Dang VT, Deng Y, Jury J, et al. Transplantation of fecal microbiota from patients with irritable bowel syndrome alters gut function and behavior in recipient mice. Sci Transl Med. 2017;9:eaaf6397.

76. Bailey MT, Dowd SE, Galley JD, Hufnagle AR, Allen RG, Lyte M. Exposure to a social stressor alters the structure of the intestinal microbiota: implications for stressor-induced immunomodulation. Brain Behav Immun. 2011;25:397-407.

77. Mirjana RS, Elena B, Heilig HGHJ, Kajsa K, Kekkonen RA, Sebastian T, et al. Global and deep molecular analysis of microbiota signatures in fecal samples from patients with irritable bowel syndrome. Gastroenterology. 2011;141:1792-801.

78. Xu D, Wu X, Grabauskas G, Owyang C. Butyrate-induced colonic hypersensitivity is mediated by mitogen-activated protein kinase activation in rat dorsal root ganglia. Gut. 2013;62:1466-74. https://doi.org/10.1136/ gutjnl-2012-302260.

79. Bourdu S, Dapoigny M, Chapuy E, Artigue F, Vasson M-P, Dechelotte $P$, et al. Rectal instillation of butyrate provides a novel clinically relevant model of noninflammatory colonic hypersensitivity in rats. Gastroenterology. 2005. https://doi.org/10.1053/j.gastro.2005.03.082.

80. Kannampalli P, Shaker R, Sengupta JN. Colonic butyrate- algesic or analgesic? Neurogastroenterol Motil. 2011. https://doi.org/10.1111/j.13652982.2011.01775.x

81. Burokas A, Arboleya S, Moloney RD, Peterson VL, Murphy K, Clarke G, et al. Targeting the microbiota-gut-brain Axis: prebiotics have anxiolytic and antidepressant-like effects and reverse the impact of chronic stress in mice. Biol Psychiatry. 2017;82:472-87. https://doi.org/10.1016/j.biopsych.2016.12.031.

82. Francis CY, Morris JW, Horwell PJ. The irritable bowel severity scoring system: a simple method of monitoring irritable bowel syndrome and its progress. Aliment Pharmacol Ther. 1997;11:395.

83. Tang YR, Yang WW, Liang ML, Xu XY, Wang MF, Lin L. Age-related symptom and life quality changes in women with irritable bowel syndrome. World Gastroenterol. 2012;18:7175-83.
84. Koloski NA, Jones M, Talley NJ. Evidence that independent gut-to-brain and brain-to-gut pathways operate in the irritable bowel syndrome and functional dyspepsia: a 1-year population-based prospective study. Aliment Pharmacol Ther. 2016:44:592-600.

85. Zimmerman M, Martin J, Clark H, Mcgonigal P, Harris L, Holst CG. Measuring anxiety in depressed patients: a comparison of the Hamilton anxiety rating scale and the DSM-5 anxious distress Specifier interview. J Psychiatr Res. 2017:93:59-63.

86. Mattiello F, Verbist B, Faust K, Raes J, Shannon WD, Bijnens L, et al. A web application for sample size and power calculation in case-control microbiome studies. Bioinformatics. 2016;32:2038-40.

\section{Publisher's Note}

Springer Nature remains neutral with regard to jurisdictional claims in published maps and institutional affiliations.

\section{Ready to submit your research? Choose BMC and benefit from:}

- fast, convenient online submission

- thorough peer review by experienced researchers in your field

- rapid publication on acceptance

- support for research data, including large and complex data types

- gold Open Access which fosters wider collaboration and increased citations

- maximum visibility for your research: over $100 \mathrm{M}$ website views per year

At BMC, research is always in progress.

Learn more biomedcentral.com/submissions 